\title{
ELECTRO-HYDROSTATIC COMPACT DRIVES WITH VARIABLE TRANSMISSION RATIO
}

\author{
Giacomo Kolks*, Jürgen Weber \\ Institut of Mechatronic Engineering, Technische Universität Dresden, Helmholtzstrasse 7a, 01069 Dresden \\ * Corresponding author: Tel.: +49 351 46339932; E-mail address: giacomo.kolks@tu-dresden.de
}

\section{ABSTRACT}

Electro-hydrostatic compact drives are an emerging technology within a range of industrially available translational drive solutions, combining the specific advantages of hydraulic and electromechanical screw drives. Compared to electromechanical screw drives, hydrostatic drives can vary their transmission ratio with comparably little effort, giving them the key advantage of downsizing the electric drive components for a given load cycle.

This paper provides a guideline on how to calculate the downsizing potential of electric motors and inverters arising from variable transmission ratio based on the load regime of a given application. Furthermore, a comprehensive systematisation of the actual switching process is described for systems that are switched by means of switching valves. The presented set of methodology is applied to demonstrators in order to validate the general findings.

Keywords: electro-hydrostatic compact drive, variable transmission, switching hydraulics

\section{INTRODUCTION}

Machine manufacturers are increasingly in demand of modular and self-contained drive solutions with high energy efficiency - without abandoning the traditional benefits of hydraulic cylinder drives, such as robustness and high force density. Electro-hydrostatic compact drives (ECD) provide substantial benefits in these aspects and are therefore an emerging technology in the hydraulic sector. ECD are displacement controlled cylinder drives with variable-speed pumps in a compact unit with an enclosed oil circuit. Alongside the advantage of improved ease-of-use compared to conventional hydraulic systems, ECD come with high robustness, high force capability and freely implementable fail-safe-behaviour compared to electro-mechanical screw drives (EMD). ECD are therefore primed to be a competitor to EMD, which have been replacing classical distributed hydraulic systems in many applications over the past years, mainly for modularisation reasons.

ECD with double-acting cylinders can generally be categorised in open hydrostatic circuit $(\mathrm{OHC})$ and closed hydrostatic circuit (CHC). $\mathrm{OHC}$ refers to structures that connect one chamber of the cylinder to the baseline pressure of a reservoir or accumulator, while the other chamber is hydrostatically connected with one pump port. In order to create a system capable of operation in four quadrants of load, typically electric or hydraulic actuated valves are needed, see Figure 1a. $\mathrm{CHC}$ on the other hand means that both cylinder chambers are constrained with both pump ports, creating a closed kinematical chain, as depicted in Figure 1b. A requirement for $\mathrm{CHC}$ is a pump structure whose flow asymmetry matches the cylinder area ratio. Furthermore, suction valves are needed in order to define the minimum pressure in the circuit.

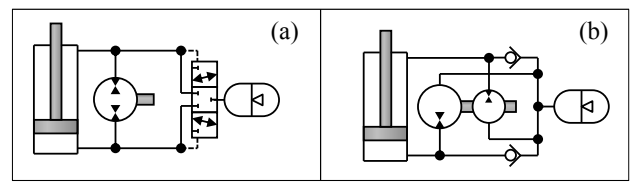

Figure 1: Exemplary circuitry structures for $\mathrm{OHC}$ with inverse shuttle valve (a) and $\mathrm{CHC}(\mathrm{b})$ drive solutions

In addition to the previously mentioned benefits of hydraulic cylinder drives compared to EMD, hydraulic circuits allow for variable transmission ratio by either varying effective pump displacement or effective cylinder area. This effect can be 
conceived as variable pitch of a screw, if the analogy to electromechanical drives is sought [1]. In applications with a distinct separation between high load and rapid traverse strokes, such as hydraulic presses, variable transmission ratio enables downsizing of the electric drive. This effect is illustrated in Figure 2 by means of single operation points: Assuming fixed transmission ratio, for speed stroke and force stroke the motor needs to be able to deliver both high rotational speed and high torque at different times of the cycle. If high force is only requested at low speed, by shifting to a higher transmission ratio the operation point of force stroke can be shifted towards high rotational speed and low torque. Thus, a motor with a lower specified torque can be used.

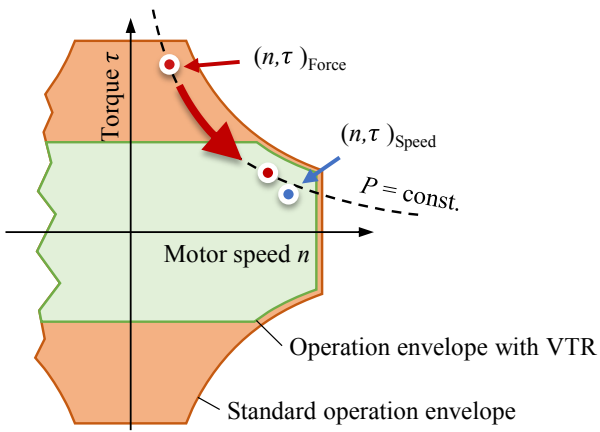

Figure 2: Illustration of motor downsizing through variable transmission ratio

It has previously been stated that in order to alter the transmission ratio, either variation of effective pump displacement (pump-based switching) or variation of effective cylinder area (actuatorbased switching) can be applied. Pump-based switching can either be achieved by physically changing the displacement of a variable pump (continuous variation), or by combining multiple fixed displacement units by means of valves and manipulating output flow by activating or deactivating flows of single units (discrete variation). Actuator-based switching lacks the possibility of continuous variation of cylinder area for technological reasons, thus only variation of effective cylinder area by means of combination of different areas of a cylinder is possible (discrete variation). The resulting solution space of ECD with variable transmission ratio is described in Table 1. With reference to the system structure it can further be refined as to whether the individual transmission stages are implemented in $\mathrm{OHC}$ or CHC. Solutions with discrete pump-based switching comprise a minimum of two pumps, solutions with discrete actuator-based switching at least to cylinder chambers.

The research project, which the paper at hand is based on, concentrated on ECD with at least one transmission stage in $\mathrm{CHC}$ using single rod cylinders. This configuration cannot be achieved with a single variable displacement pump, leading to neglecting continuously variable solutions.

Table 1: Solution space of ECD with two transmission stages

\begin{tabular}{c|c|c|c}
\hline & \multicolumn{2}{|c|}{ pump-based switching } & $\frac{\text { actuator- }}{\text { based }}$ \\
$\begin{array}{c}\text { circuit type } \\
\mathrm{CHC} / \mathrm{OHC}\end{array}$ & continuous & discrete & $\begin{array}{c}\text { discrete } \\
\text { ditching }\end{array}$ \\
\hline $\begin{array}{c}\text { one stage } \mathrm{OHC} / \\
\text { one stage } \mathrm{CHC}\end{array}$ & $\mathrm{PC} 1$ & $\mathrm{PD} 1$ & $\mathrm{AD} 1$ \\
\hline $\begin{array}{c}\text { both stages } \mathrm{CHC} \\
\text { bothes } \mathrm{OHC}\end{array}$ & $\mathrm{PC} 2$ & $\mathrm{PD} 2$ & $\mathrm{AD} 2$ \\
\hline
\end{tabular}

Creating solutions with $\mathrm{CHC}$ structure in both transmission stages is a design problem within tight constraints concerning pump displacements and cylinder areas. A comprehensive approach to describe the subspace of these solutions is given by the authors for discrete pump-based switching [2] and discrete actuator-based switching [3], as well as state-of-the-art in switching linear drives.

Pioneer work on displacement controlled cylinder drives has been carried out by Sprockhoff [4] and Berbuer [5], dealing with OHC structures and valves to ensure a minimum pressure level in the cylinder and oil exchange with a reservoir. Important foundations for asymmetric single rod cylinders with displacement control in $\mathrm{CHC}$ structure have been laid by Lodewyks [5]. More recent research by Michel examined both $\mathrm{OHC}$ and $\mathrm{CHC}$ types, proving that $\mathrm{OHC}$ tend to be more energy efficient, while $\mathrm{CHC}$ having the better controllability $[6,7]$. It has been shown that $\mathrm{OHC}$ structures with inverse shuttle valves exhibit discontinuities and instabilities when the load pressure is around zero or changes directions. Instability issues through pressure-piloted switching valves in this context have been investigated in further research $[8,9]$. A good overview over the current state of the art in ECD is included in the work by Ketelsen et al. [10]. 


\section{DOWNSIZING POTENTIAL}

\subsection{Dimensioning of electric drivetrain}

Choice of electric motors and servo inverters are crucial to ECD drives, since they are strongly related with their static and dynamic properties as well as total costs. In the following, dimensioning is carried out with relation to typical combinations of servo inverters and synchronous servo motors.

Usually, servo motors are dimensioned so that they withstand the thermal load over a specific cycle and the maximum dynamic torque that occurs over time. The maximum torque criterion (MTC) can be formulated as the following:

$\max (\tau(t))<\tau_{\mathrm{M} \text {,max }}$

As a thermal criterion, a motor exhibits an S1line $\tau_{\mathrm{M} 1}(n)$ in the torque-speed plane, which has to lie towards the outside of operating point of equivalent load $\left(n_{\mathrm{eq}}, \tau_{\mathrm{eq}}\right)$ of the motor during its load cycle. It is often an acceptable simplification to assume the S1-curve to be parallel to the speed axis, thus for generalisation purposes this will apply. The torque value of the horizontal S1-line will be referred to as the nominal torque $\tau_{\mathrm{N}}$. Thus, the thermal criterion (equivalent torque criterion, ETC) reads

$\tau_{\text {eq }}<\tau_{\mathrm{M}, \mathrm{nom}}$

with the equivalent torque

$\tau_{\text {eq }}=\sqrt{\frac{1}{T} \int_{T} \tau(t)^{2} \mathrm{~d} t .}$

This equivalent torque formulation correlates the electric losses of the motor to the square of the torque according to the following:

$P_{\text {loss }}(t) \propto I(t)^{2} \propto \tau(t)^{2}$

Any electric motor has to fulfil both maximum torque and equivalent torque criterion that are calculated based on the torque regime $\tau(t)$ of the load cycle.

\subsection{Choice of transmission ratios}

ECD can be regarded as transmission that transform rotational mechanical input power into translational mechanical output power. The transmission ratio can be defined as

$i=\frac{\omega}{\dot{x}}=\frac{2 \pi n}{\dot{x}}$

Neglecting losses, conservation of energy results in

$$
i=\frac{F}{\tau} \text {. }
$$

For the simplified and idealised ECD structure shown in Figure 3, the transmission ratio yields

$i=\frac{2 \pi A_{\mathrm{eff}}}{V_{\mathrm{P}, \mathrm{eff}}}$.

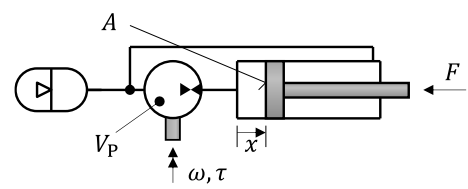

Figure 3: Simplified structure of ECD

It is obvious that, by changing effective cylinder area $A_{\text {eff }}$ or effective pump displacement volume $V_{\mathrm{P}, \text { eff }}$, the transmission ratio can be varied.

Let $i^{\mathrm{F}}$ be the transmission ratio in force stage, and $i^{\mathrm{S}}$ the transmission ratio in speed stage of any drive with variable transmission ratio. The transmission range is defined as

$\alpha_{i}=\frac{i^{\mathrm{F}}}{i^{\mathrm{S}}}$

and from eqs. (5)-(6) we can derive

$\alpha_{i}=\frac{\tau^{\mathrm{S}}(F)}{\tau^{\mathrm{F}}(F)}=\frac{n^{\mathrm{F}}(\dot{x})}{n^{\mathrm{S}}(\dot{x})}$.

For calculation of downsizing potential, following hypothesis is used:

The size of an electric servo drive is minimal if the required torque is minimal.

Thus, a drive dimensioning minimises the drive size, whenever it maximises the rotational speed. In direct-driven hydraulics, pumps are typically the limiting factor of rotational speed, thus the goal is to reach the maximum pump speed in both force and speed stage:

$\left|n^{\mathrm{S}}\right|_{\max }=\left|n^{\mathrm{F}}\right|_{\max }=n_{\text {max }}$

Neglecting leakage, the rotational speed is

$n(t)=\left\{\begin{array}{l}\frac{i^{\mathrm{S}}}{2 \pi} \cdot \dot{x}(t), \text { in speed stage } \\ \frac{i^{\mathrm{F}}}{2 \pi} \cdot \dot{x}(t), \text { in force stage. }\end{array}\right.$

Thus, an optimal transmission range can be derived as the ratio of maximum speed in speed stage over maximum speed in force stage:

$\alpha_{i}=\frac{\left|\dot{x}^{\mathrm{S}}\right|_{\max }}{\left|\dot{x}^{\mathrm{F}}\right|_{\max }}$ 
Neglecting friction losses, the torque yields

$$
\tau(t)=\left\{\begin{array}{l}
\frac{F(t)}{i^{\mathrm{S}}}, \text { in speed stage } \\
\frac{F(t)}{i^{\mathrm{F}}}, \text { in force stage. }
\end{array}\right.
$$

\subsection{Downsizing potential for electric motors}

For a reference, an ECD with fixed transmission ratio is dimensioned in a way that it reaches the maximum pump speed. The equivalent torque is

$\tau_{\text {eq,Ref }}=\sqrt{\frac{1}{T_{\text {tot }}} \int_{T_{\text {tot }}}\left(\frac{F(t)}{i_{\text {Ref }}}\right)^{2} \mathrm{~d} t}$

while maximum torque yields

$\tau_{\text {max,Ref }}=\frac{F_{\text {max }}}{i_{\text {Ref }}}$.

The load cycle is divided in a speed stage $T_{\mathrm{S}}$ and a force stage $T_{\mathrm{F}}$, see Figure 4, and the transmission of the switching drive is chosen accordingly.

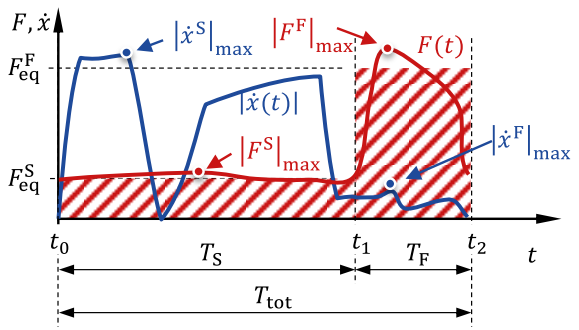

Figure 4: Exemplary machine cycle

According to the findings in eq. (12) and an optimally dimensioned reference transmission ratio, following transmission ratios can be assumed:

$i_{\text {Ref }}=i^{\mathrm{S}}=\frac{i^{\mathrm{F}}}{\alpha_{i}}$

With this definition, we can calculate the ratio between the equivalent torque of the reference drive over the equivalent torque of the switching drive $\alpha_{\tau, \mathrm{eq}}:$

After introducing proportional parameters

$\alpha_{F}=\frac{F_{\mathrm{eq}}^{\mathrm{F}}}{F_{\mathrm{eq}}^{\mathrm{S}}}$

$\alpha_{t}=\frac{T_{\mathrm{F}}}{T_{\mathrm{S}}}$
$F_{\text {eq }}=\sqrt{\frac{1}{T} \int_{T} F(t)^{2} \mathrm{~d} t}$

the equivalent torque ratio results in

$\alpha_{\tau, \text { eq }}=\frac{\tau_{\text {eq }}}{\tau_{\text {eq, Ref }}}=\sqrt{\frac{1+\frac{\alpha_{F}^{2} \cdot \alpha_{t}}{\alpha_{i}^{2}}}{1+\alpha_{F}^{2} \cdot \alpha_{t}}}$.

The ratio of maximum torques is

$\alpha_{\tau, \text { max }}=\frac{\tau_{\text {max }}}{\tau_{\text {max }, \text { Ref }}}=\min \left(\alpha_{i}, \frac{\left|M^{\mathrm{E}}\right|_{\text {max }}}{\left|M^{\mathrm{K}}\right|_{\max }}\right)$.

With these parameters $\alpha_{\tau \text {,eq }}, \alpha_{\tau, \max }$ an assessment of downsizing potential can be made. If both reference fixed drive and variable transmission drive are limited by the equivalent torque criterion, the downsizing parameter concerning rated torque is equal to the equivalent torque ratio $\alpha_{\tau, \text { eq }}$. However, this is not always the case, thus differentiation has to be made.

Whether a motor is limited by maximum torque or equivalent torque depends on its overload capacity, meaning the ratio of maximum torque to nominal torque:

$\alpha_{\mathrm{OL}}=\frac{\tau_{\mathrm{Mot}, \mathrm{max}}}{\tau_{\text {Mot,nom }}}$

For generalisation, we assume that all feasible motors that are within the choice range share the same overload capacity $\alpha_{\mathrm{OL}}$. Under this circumstance, downsizing factors with respect to torque specifications are listed in Table 2. The downsizing factor means the ratio of reduction of required nominal motor torque that can be achieved by means of transmission switching against a fixed transmission reference drive:

$\alpha_{\tau, \mathrm{Mot}}=\frac{\tau_{\mathrm{M}, \mathrm{nom}}}{\tau_{\mathrm{M}, \mathrm{nom}, \mathrm{Ref}}}$

The resulting calculation schedule for downsizing potential is thus:

1. Choose force stage intervals and speed stage intervals according to the load cycle.

2. Calculate transmission range with eq. (12) and $\alpha_{\tau, \text { eq }}$ using eqs. (17)-(20).

3. Choose a realistic overload capacity $\alpha_{\mathrm{OL}}$

4. Select case and calculate downsizing factor $\alpha_{\tau, \text { Mot }}$ according to Table 2 .

and with definition of an equivalent force 
Table 2: Calculation of downsizing factors depending on load conditions

\begin{tabular}{|c|c|c|c|}
\hline & Limiting criteria & Condition & Downsizing factor \\
\hline Case 1 & $\begin{array}{l}\text { Reference } \rightarrow \text { MTC } \\
\text { Switching drive } \rightarrow \text { MTC }\end{array}$ & $\frac{F_{\max }}{\alpha_{i} \cdot F_{\text {eff }} \cdot \alpha_{\tau, \mathrm{eq}}}>\alpha_{\mathrm{OL}}$ & $\alpha_{\tau, \mathrm{Mot}}=\alpha_{\tau, \max }$ \\
\hline Case 2 & $\begin{array}{l}\text { Reference } \rightarrow \text { MTC } \\
\text { Switching drive } \rightarrow \text { ETC }\end{array}$ & $\frac{F_{\mathrm{max}}}{F_{\mathrm{eff}}}>\alpha_{\mathrm{OL}}>\frac{F_{\mathrm{max}}}{\alpha_{i} \cdot F_{\mathrm{eff}} \cdot \alpha_{\tau, \mathrm{eq}}}$ & $\alpha_{\tau, \mathrm{Mot}}=\frac{\alpha_{\mathrm{OL}} \cdot F_{\mathrm{eff}} \cdot \alpha_{\tau, \mathrm{eq}}}{F_{\max }}$ \\
\hline Case 3 & $\begin{array}{l}\text { Reference } \rightarrow \text { ETC } \\
\text { Switching drive } \rightarrow \text { ETC }\end{array}$ & $\alpha_{\mathrm{OL}}>\frac{F_{\mathrm{max}}}{F_{\mathrm{eff}}}$ & $\alpha_{\tau, \mathrm{Mot}}=\alpha_{\tau, \mathrm{eq}}$ \\
\hline
\end{tabular}

Switching transmission ratio also has an effect on the choice of servo inverters. These are often dimensioned to withstand the maximum current during the cycle, which correlates with maximum torque. Thus, the downsizing factor can be considered to be

$\alpha_{\mathrm{I}, \mathrm{Inv}}=\alpha_{\tau, \max }$

This methodology is applied to an idealised cycle of a hydraulic press brake in the following. The cycle data is given in Figure 5 (left) by means of cylinder position and speed as well as load force curves over time. The resulting motor torque and speed are shown in the diagram in the middle of the same figure, for a reference drive with fixed transmission and an ECD with variable transmission. Switching is carried out at the times $t_{a}$ and $t_{b}$. By evaluating the cycle in the described manner, the downsizing factor of the motor results in $\alpha_{\tau, \mathrm{Mot}}=0.22$ (case 3) and $\alpha_{\mathrm{I}, \mathrm{Inv}}=$ 0.19 for the inverter. Using an exemplary model range of synchronous servo motors and inverters in the power range of 1 to $10 \mathrm{~kW}$, the benefits of variable transmission in terms of weight and cost savings are given in Figure 5 on the right.

\section{DEMONSTRATOR DRIVES}

In order to validate the findings from the project, two demonstrator ECD that arose from the systematic scan of solution space $[3,2]$ have been set up - one for each switching principle - as depicted in Figure 6.

The pump-based switching demonstrator (PBD) makes use of a $\mathrm{CHC}$ structure in speed
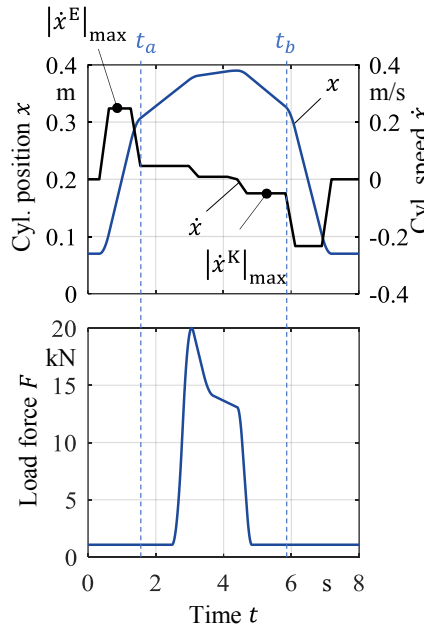
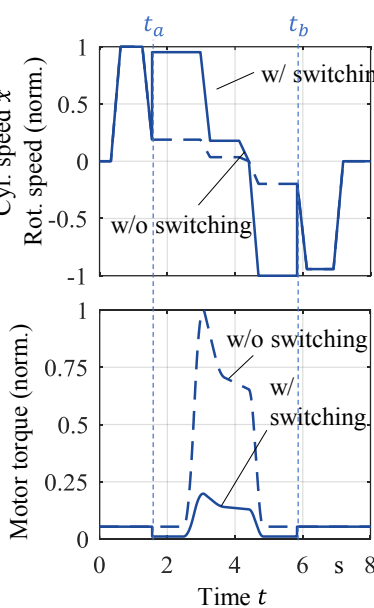

Downsizing Potential

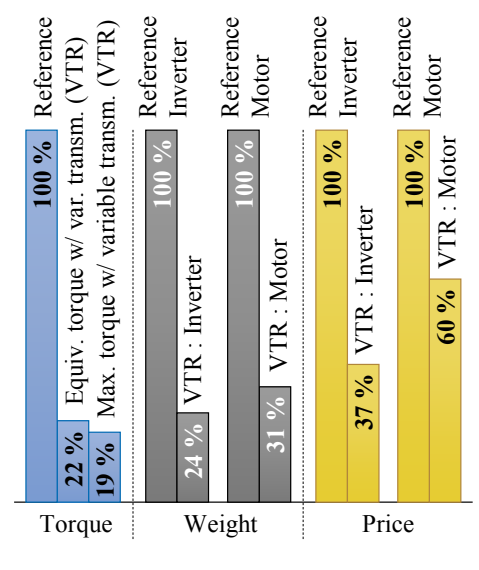

Figure 5: Cycle of an idealised press brake with motor torque and speed with and without switching transmission, and downsizing in terms of weight and cost of exemplary commercially available component. 


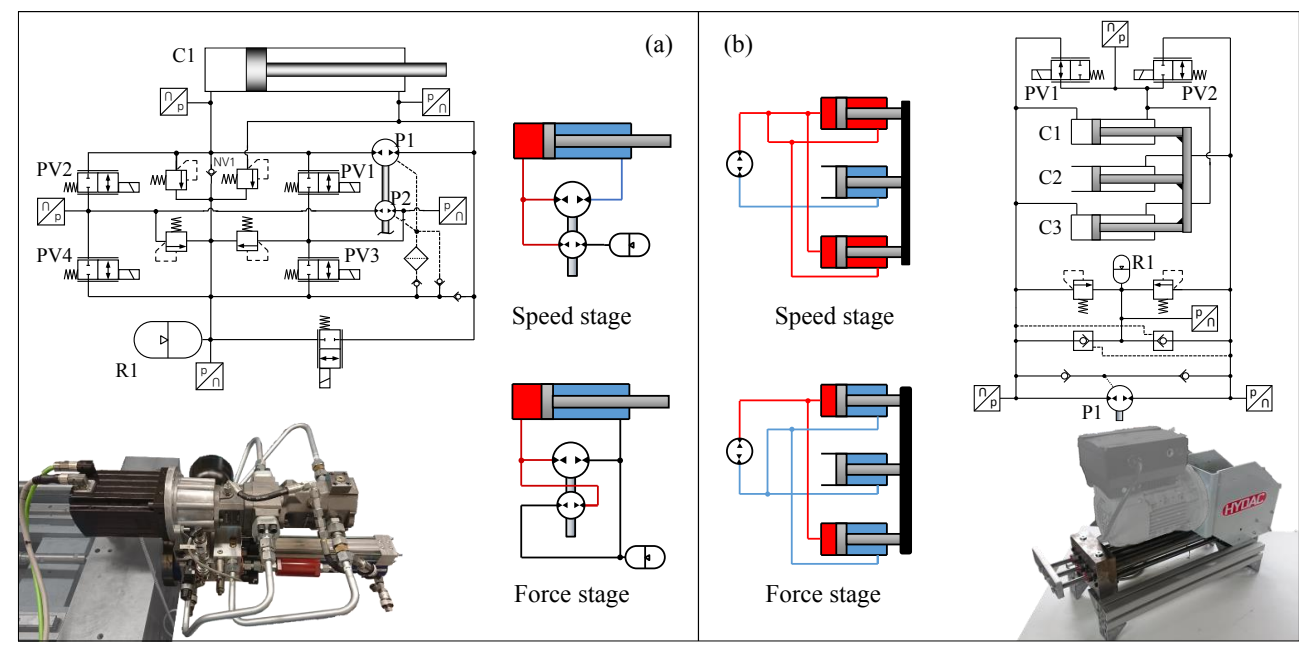

Figure 6: Demonstrators PBD (a) and ABD (b)

stage, where the sum of two pump outflows is connected to the head-side cylinder chamber while the rod-side chamber receives the flow only from the larger pump. Due to $\mathrm{CHC}$, the ratio of pump displacement volumes has to be related to the cylinder area ratio. In force stage, an $\mathrm{OHC}$ is applied such, that the rod-side chamber is connected with the reservoir. The head side chamber receives a flow that results from the difference of both pump outflows. Torque reduction is due to one unit working in pump mode, and the other working in motoring mode on the same shaft. Switching is carried out via five $2 / 2$ directional proportional solenoid cartridge valves. For details see [2].

The actuator-based switching demonstrator (ABD) comprises three parallel, identical single rod cylinders with mechanically coupled piston rods. In force stage, one pump port acts on the three rod-side chambers, while the other pump port acts on the two outer head-side chambers. The centre head-side chamber is constantly vented. In speed stage, the two outer rod side chambers are switched to the other pump port. Since the cylinders are dimensioned in a way that the cylinder unit acts as a statically symmetrical consumer in both stages, it can be operated in $\mathrm{CHC}$ with a single pump. However, in order to avoid any undesired pressure increase, pilot operated check valves are used to define the minimum pressure in the used system. Switching valves are two valves identical to the ones used in PBD. More details on this demonstrator can be found in [3].
In terms of the systemisation shown in Table 1, PBD can be considered PC1 and ABD can be considered AD2.

Significant parameters for both systems are listed in Table 3.

Table 3: Demonstrator dimensioning parameters

\begin{tabular}{lll}
\hline & PBD & ABD \\
\hline Pump dis- & $V_{\mathrm{P} 1}=10,8 \mathrm{~cm}^{3}$ & $V_{\mathrm{P} 1}=5 \mathrm{~cm}^{3}$ \\
placement & $V_{\mathrm{P} 2}=6,9 \mathrm{~cm}^{3}$ & \\
Cylinder & $\varnothing(40 \mathrm{~mm} / 25 \mathrm{~mm})$ & $\varnothing(25 \mathrm{~mm} / 14,43 \mathrm{~mm})$ \\
dimensions & x $450 \mathrm{~mm}$ & $\mathrm{x} 400 \mathrm{~mm}$ \\
Reservoir & $V_{\text {tot }}=1,4 \mathrm{l}$ & $V_{\text {tot }}=80 \mathrm{ml}$ \\
transm. range & $1: 4.5$ & $1: 3$ \\
\hline
\end{tabular}

\section{SWITCHING STRATEGY}

The aim of the switching process is to change the transmission. Obviously, a discontinuous switch to a different transmission ratio will lead to a disturbance of the system, similar to a step in the primary command signal of the prime mover.

In order to allow a smooth transition, valve trajectories are generated such that the transmission ratio is a smooth curve from start to end of the process. Further we demand

$i^{\mathrm{F}} \geq \frac{\omega(t)}{\dot{x}(t)} \geq i^{\mathrm{S}}$

throughout the switching process. Since due to valve opening, the drive is no longer a hydrostatic transmission, the load has to be taken into account. 


\subsection{Systematisation}

In previous work $[3,2]$, the definition of a transmission stage allocates each pump port to any cylinder port or the reservoir port in case of pump-based switching, or allocates each cylinder port to any pump port or the reservoir in case of actuator-based switching, respectively. Thus, any switching process can be thought of as a sequence of reallocations of pump ports (pump-based switching) or cylinder ports (actuator-based switching). Reallocation of one port is called an elementary switching process (ESP), which is illustrated in Figure 7.

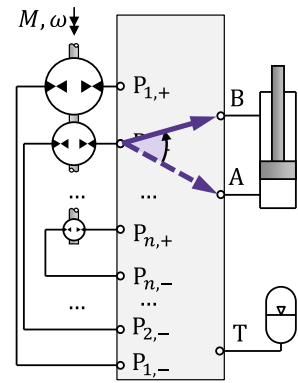

Pump-based switching

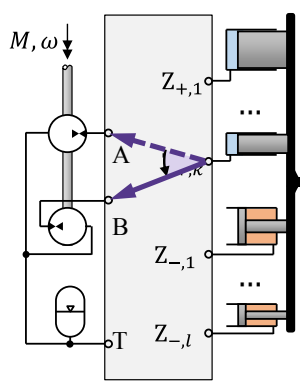

Actuator-based switching
Figure 7: Examples of elementary switching processes

Any such ESP, of which global switching processes are constituted, have the logic of an electric two-way switch, or a 3/2-directional valve. A general way of realisation with one more degree of freedom is a combination of two 2/2-directional valve per ESP, as shown in Figure 8.

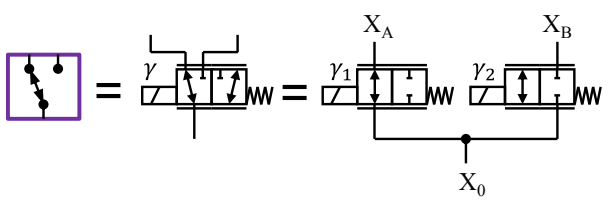

Figure 8: Valve architectures for ESP

The advantage of separating the switching process in ESP is unification of control strategies. Since all ESP can be treated similarly, even complex global switching processes can be broken down to manageable units.

The meaning of ESP in terms of the demonstrator drives is given in Figure 9. For PBD, there are two ESP that have to be switched consecutively. Between the two ESP, there is an intermediate state, in which the smaller pump idles at negligible pressure difference. The exact order, in which ESP1 and ESP2 are switched decide on whether the pump idles at the pressure level of the reservoir or of the cylinder's head-side chamber. Before any switching is carried out, PV5 has to be opened in order to open the hydrostatic circuit on the rod-side chamber to reservoir. In case of $\mathrm{ABD}$, only one ESP is required.

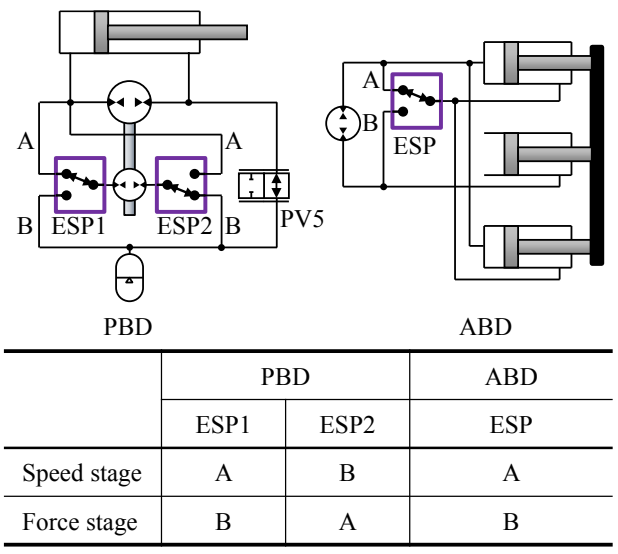

Figure 9: ESP in demonstrator drives and state table

With reference to the nomenclature in Figure 6, ESP1 of PBD consists of valves PV2 and PV4, while ESP2 consists of PV1 and PV3. As to ABD, ESP obviously consists of PV1 and PV2.

\subsection{Elementary switching process}

Although all ESP have the same structure, the boundary conditions do vary between pumpbased and actuator-based switching. With reference to the port labels in Figure 8, pump-based ESP can be considered to have an independent flow input at port $\mathrm{X}_{0}$ due to exclusively having one pump port directly attached, and independent pressure input at port $\mathrm{X}_{\mathrm{A}}$ and $\mathrm{X}_{\mathrm{B}}$ depending on load. When considering actuator-based switching, flows and pressure on all ports depend on interaction of loads, pump speeds and valve positions. However, strategies developed for the simpler pump-based switching proved to work for actuator-based switching as well.

Modelling of ESP is carried out based on the system depicted in Figure 11. It is governed by turbulent valve flow and flow continuity:

$Q_{0}=Q_{\mathrm{A}}+Q_{\mathrm{B}}$

$Q_{\mathrm{A}}=B \cdot \gamma_{\mathrm{A}} \cdot \operatorname{sgn}\left(p_{0}-p_{\mathrm{A}}\right) \cdot \sqrt{\left|p_{0}-p_{\mathrm{A}}\right|}$

$Q_{\mathrm{B}}=B \cdot \gamma_{\mathrm{B}} \cdot \operatorname{sgn}\left(p_{0}-p_{\mathrm{B}}\right) \cdot \sqrt{\left|p_{0}-p_{\mathrm{B}}\right|}$ 
$B$ is the turbulent flow coefficient that accounts for nominal valve flow at a given pressure difference.

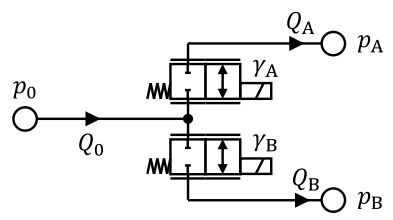

Figure 11: ESP model

Control strategies for the proportional valves of an ESP are based on a static analysis of the switching process with the boundary conditions of pump-based switching at constant motor speed and constant load. An exemplary analysis under such constraints is given in Figure 10 in the plane over the two normalized openings of the valves involved. Any switching process requires a transition from point $\mathrm{X}$ to $\mathrm{Y}$ or vice versa across the plane. Any trajectory through the plane results in increased loss over the valve control edges. Every load and speed condition exhibits a saddle point of minimum unavoidable power loss, marked with ' $M$ ' in the given example.

Choosing a bilinear valve trajectory through the saddle point ' $M$ ' as shown in Figure 10 minimises losses and fulfils eq. (25). Moving the corner point further towards the upper right results in flow losses (short-circuit flow across the valves $\left.\left(Q_{\mathrm{A}}<0\right)\right)$ and thus violates eq. (25). Moving it further down towards lower $\gamma_{\mathrm{B}}$ results in drastic pressure increase and thus torque loss. At negative $Q_{0}$ it would even lead to serious cavitation.

With such valve trajectories, the challenge is to meet accuracy requirements using simple direct solenoid driven valves without spool position feedback. Around point ' $\mathrm{M}$ ' the pressure $p_{0}$ is sensitive to valve spool position, leading to dynamic excitation of the system especially at actuator-based switching.

A more robust strategy therefore makes use of a $p_{0}$ pressure feedback to 'guide' one valve control signal along it correct trajectory. An overview of the strategy is given in the following, with reference to Figure 10:

Depending on flow direction of $Q_{0}$ and direction of pressure difference across the switch $p_{A}-$ $p_{\mathrm{B}}$, the saddle point ' $\mathrm{M}$ ' lies on one of the axes $\gamma_{\mathrm{A}}$ or $\gamma_{\mathrm{B}}$. As a characteristic property of the proposed control strategy, the valve command signal on whose axis ' $\mathrm{M}$ ' is located, is governed by a pressure feedback controller. In the example given in Figure 10, this is signal $\gamma_{\mathrm{B}}$. For the exemplary transition from point $\mathrm{X}$ to point $\mathrm{Y}$, the control strategy is given in Figure $\mathbf{1 2}$ by means of state chart notation.

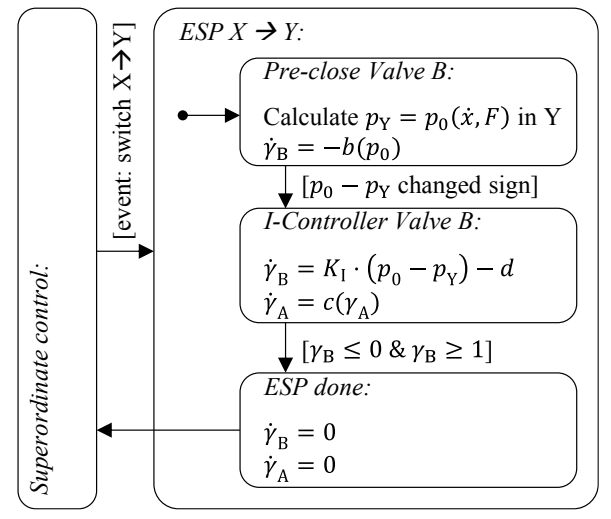

Figure 12: ESP sequence $X \rightarrow Y$, see Figure 10

First, the valve $\mathrm{B}$ is closed using a trajectory that takes $p_{0}$ into account in order to prevent overshoot. During that process, the pressure $p_{0}$ corresponding to point ' $\mathrm{Y}$ ' is constantly calculated based on measurement data. Once $p_{0}$ reaches that
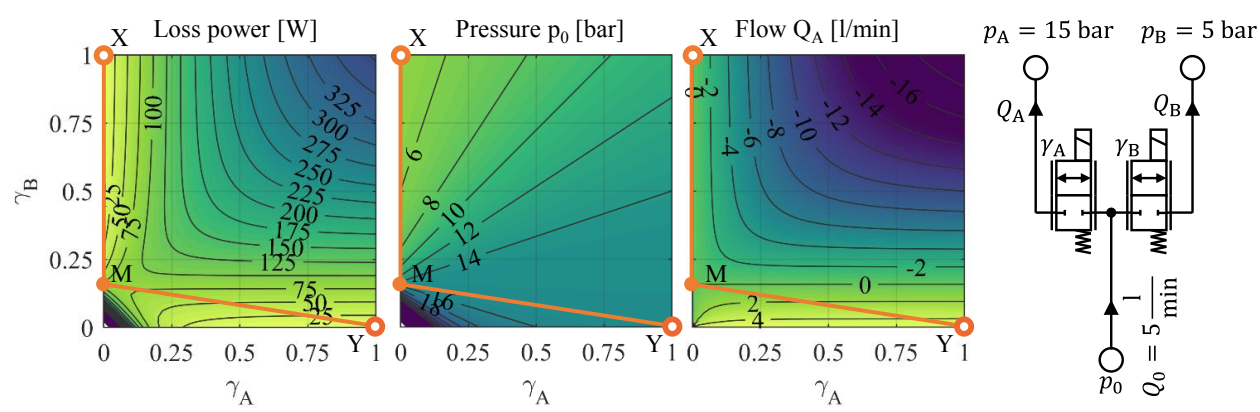

Figure 10: Static behaviour of the ESP for exemplary boundary conditions 
pressure $p_{\mathrm{Y}}$, the integral pressure feedback controller is activated to $\operatorname{keep} p_{0}$ at $p_{\mathrm{Y}}$ while opening valve $\mathrm{A}$. The term $d$ in the pressure feedback control law makes sure that valve B closes and the switching process carries on. When the conditions of point ' $\mathrm{Y}$ ' are met in terms of valve command signals, the ESP is completed. The parameters or functions $K_{\mathrm{I}}, b, c, d$ have an impact on the smoothness and duration of the ESP, however an optimal set is yet to be defined and depend strongly on the dynamic properties of the system and valves.

\section{VALIDATION}

The pump-based switching demonstrator is used to validate both the switching strategy and the downsizing potential on a load test stand. In the following, a range of experimental results will be presented. Results using ABD remain subject of future publications.

\subsection{Switching strategy}

The first example shown is a switching procedure from speed stage to force stage at constant motor speed of $n=1000 \mathrm{~min}^{-1}$ for different load forces, with result plotted in Figure 13. The cylinder velocity at low load force $(F=1 \mathrm{kN})$ took a smooth decline over the course of switching, regardless of whether the smooth switching strategy was applied $(\Delta t=0.25 \mathrm{~s})$ or whether all valves were operated at the same time and as fast as possible $(\Delta t=0 \mathrm{~s})$. The smooth transition at discrete switching operation may be due to low response of the valve, but cannot be verified since spool position measurement is not implemented.

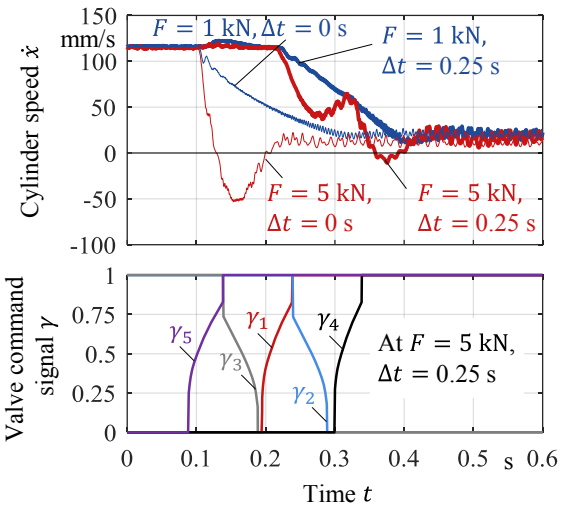

Figure 13: Switching from speed stage to force stage at $n=1000 \mathrm{~min}^{-1}$ at constant load force $F$
At higher load, the smooth switching strategy yields a smoother transition and less overshoot than discrete switching. When all valves are actuated at one time, uncontrolled short-circuit flow makes the mass accelerate in direction of external load, which is prevented using the above described valve trajectory.

The bottom diagram of Figure 13 shows the valve command signals during the controlled switching process. Integral-dominated pressure feedback control lets both valves be closed at the same times $(t=0.19 \mathrm{~s}$ and $t=0.29 \mathrm{~s})$ in both ESP. That would mean the flow to be blocked and dramatic pressure increase or cavitation at on pump port. The absence of these proves that the spool positions of the closing valves lack behind their command signal significantly.

A similar switching procedure was run at a motor speed that was generated by a dynamic feedforward control to keep the cylinder speed constant. The controller calculates the motor speed according to desired cylinder speed trajectories, taking plant dynamics, load force and valve positions into account. Measurement result are shown in Figure 14.

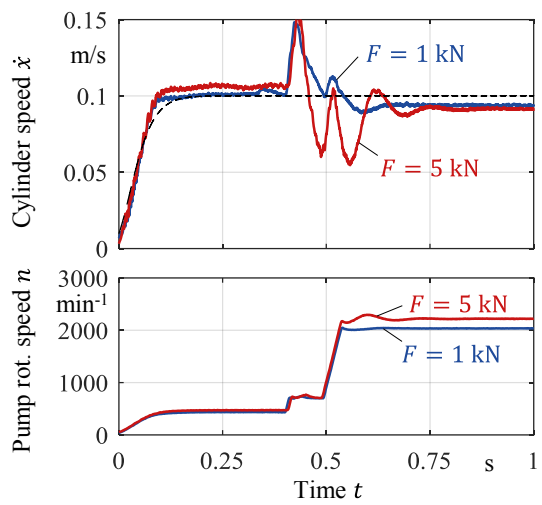

Figure 14: Switching from speed stage to force stage at constant load force $F$ and with feedforward speed control

It becomes obvious that while switching, the trajectory cannot be tracked by the motor speed command. The reason is mainly related to deviations between valve command signal and actual spool opening. The feedforward control law receives the valve command signal and calculates required rotational speeds based on these.

\subsection{Downsizing potential}

Downsizing potential of the demonstrator drive 
can be assessed by mapping the torque requirement over speed and load force in speed stage and force stage. The associated measurement results are illustrated in Figure 15. Speed stage recordings could not exceed a load force of $7 \mathrm{kN}$ in the experiment, since the used motor was restricted to a nominal torque of $20 \mathrm{Nm}$. Linear extrapolation of the curves however is admissible. A torque reduction due to force stage can clearly be observed at high load force. Given a maximum torque of $20 \mathrm{Nm}$, the achievable force in force stage is about three times the achievable force in speed stage.

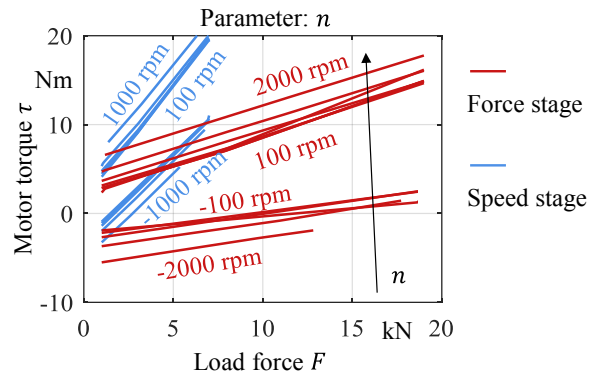

Figure 15: Measurement of torque over speed and force

As further proof of concept, the press brake cycle analysed in Figure 4 is implemented on the load test bench. The results in terms of torque are compared in Figure 16 for the demonstrator in speed stage only and with switching activated. The resulting ratios of maximum and equivalent force are $\alpha_{\tau, \max }=0.31$ and $\alpha_{\tau, \max }=0.43$ respectively, resulting in the estimated cost reduction shown in Figure 16.
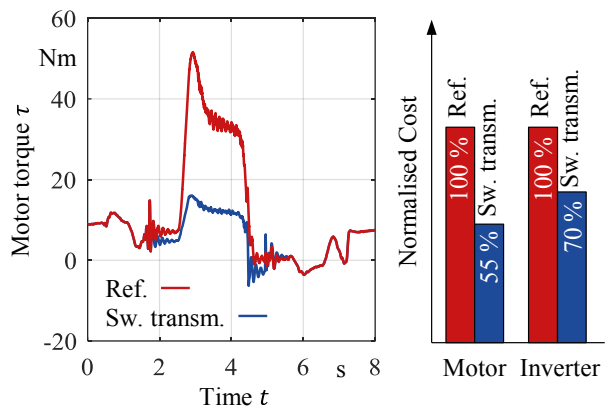

Figure 16: Measurement of torque in press brake cycle from Figure 5, for reference and switching driving

The cost reduction is lower compared to the reductions estimated under ideal conditions, as shown in Figure 5. This is mainly due to to causes: Firstly, pump friction has a major impact on motor torque in force stage and, secondly, the transmission range of the demonstrator is lower than the optimal transmission range for the chosen load cycle, due to a restricted choice of cylinder area ratios, as documented in [2].

\section{CONCLUSION AND OUTLOOK}

The paper at hand provides a guideline on how to estimate the downsizing potential of variable transmission linear drives in comparison to fixed transmission drives, depending on a given load cycle. For an exemplary press brake cycle, the maximum downsizing potential is estimated to be around $80 \%$ in terms of torque. Using a demonstrator drive, the achievable downsizing potential was reduced to $57 \%$ mainly due to pump friction. Even with friction related losses, substantial cost savings of the electric components can be realised.

A general approach to handle switching processes for discrete variable transmission drives is given and applied to the demonstrators. Switching smoothness was found to be improved with valve trajectories based on pressure feedback in comparison to discrete switching, although valve accuracy proved to be crucial to attainable switching smoothness in general. When it comes to adoption of the strategy for application in production machinery, thorough measurement and characterization of the used valves is necessary to meet accuracy specifications when switching under load occurs.

Approaches that may be investigated in future work are ballistic switching strategies that might be applied when inertia is high in comparison to external load forces.

\section{ACKNOWLEDGEMENT}

The presented research activities are part of the project "Elektrohydraulische Kompaktantriebe mit schaltbarer Übersetzung" (Ref. No. 703490). The authors would like to thank the Fluid Power Research Fund of the VDMA for the funding and support.

\section{NOMENCLATURE}

ABD Actuator-based switching demonstrator

CHC Closed hydrostatic circuit 
ECD Electro-hydrostatic compact drive

EMD Electromechanical drive

ESP Elementary switching process

ETC Equivalent torque criterion

MTC Maximum torque criterion

$\mathrm{OHC}$ Open hydrostatic circuit

PBD Pump-based switching demonstrator

VTR Variable transmission ratio

eff Effective

eq Equivalent

F Force stage

max Maximum

Mot Motor

nom Nominal

OL Overload

P Pump ports

Inv Inverter

Ref Reference

$\mathrm{S} \quad$ Speed stage

$\mathrm{T} \quad$ Reservoir port

tot Total

X Port label

$\mathrm{X}, \mathrm{Y}$ Operation points

Z Cylinder ports

A Area $\mathrm{m}^{2}$

$B \quad$ Flow coefficient

$b \quad$ control parameter $1 / s$

c control function $1 / \mathrm{s}$

$d \quad$ control parameter $\quad 1 / \mathrm{s}$

F Force N

I Electric current A

$i$ Transmission ratio -

$K_{\mathrm{I}} \quad$ Integral gain $\quad 1 / \mathrm{Pa} \mathrm{s}$

$n \quad$ Rotational speed $1 / \mathrm{s}$

$p \quad$ Pressure $\quad \mathrm{Pa}$

$P$ Power W

$Q \quad$ Flow rate $\mathrm{m}^{3} / \mathrm{s}$

$t$ Time $\mathrm{s}$

$T \quad$ Time interval s

$V_{\mathrm{P}} \quad$ Pump displacement volume $\quad \mathrm{m}^{3}$

$x \quad$ Cylinder position $\quad \mathrm{m}$

$\begin{array}{lll}\alpha & \text { Ratio parameter } & - \\ \gamma & \begin{array}{l}\text { Normalised valve command } \\ \text { signal }\end{array} & - \\ \tau & \text { Torque } & \mathrm{Nm} \\ \omega & \text { Angular speed } & \mathrm{rad} / \mathrm{s}\end{array}$

\section{REFERENCES}

[1] Brahmer, B.: On Adaptive Electro Hydrostatic Actuators. In: Proceedings of the 11th International Fluid Power Conference (11. IFK). Aachen, Germany, 2018

[2] Kolks, G.; Weber, J.: Compact Cylinder Drives with Variable Transmission Ratio Using Multiple Pumps. In: Proceedings of the 10th Workshop on Digital Fluid Power. Linz, AT, 2019

[3] Kolks, G.; Weber, J.: Symmetric Single Rod Cylinders with Variable Piston Area? A Comprehensive Approach to the Right Solution. In: Proceedings of the ASME/BATH 2018 Symposium on Fluid Power \& Motion Control : FPMC 2018. Bath, UK, 2018

[4] Sprockhoff, V.: Untersuchungen von Regelungen im hydrostatischen Zylinderantrieb mit Servopumpe. RWTH Aachen. Dissertation. 1979

[5] Berbuer, J.: Neuartige Servoantriebe mit primärer Verdrängersteuerung. RWTH Aachen. Dissertation. 1988

[6] Michel, S.; Weber, J.: Electrohydraulic compactdrives for low power applications. In: Proceedings of the 7th FPNI PhD-Symposium on Fluid Power. Reggio Emilia, Italy, 2012

[7] Michel, S.: Energieeffiziente elektrohydraulische Antriebe kleiner Leistung. Dresden, TU Dresden. Research Report. 2012

[8] Çalışkan, Hakan; Balkan, Tuna; Platin, Bülent E.: A Complete Analysis and a Novel Solution for Instability in Pump Controlled Asymmetric Actuators. In: Journal of Dynamic Systems, Measurement, and Control 137 (2015), Nr. 9, 091008091008-14

[9] Williamson, C.; Ivantysynova, M.: Stability and motion control of inertial loads with displacement controlled hydaulic actuators. In: Proceedings of the 6th FPNI PhD-Symposium on Fluid Power. West Lafayette, USA, 2010

[10] Ketelsen, Søren; Padovani, Damiano; Andersen, Torben; Ebbesen, Morten; Schmidt, Lasse: Classification and Review of Pump-Controlled Differential Cylinder Drives. In: Energies 12 (2019), Nr. 7, p. 1293 